\title{
Longevity of Supersymmetric Flat Directions
}

\author{
Rouzbeh Allahverdi ${ }^{1,2}$ and Anupam Mazumdar ${ }^{3}$ \\ 1 Perimeter Institute for Theoretical Physics, Waterloo, ON, N2L 2Y5, Canada. \\ 2 Department of Physics and Astronomy, McMaster University, Hamilton, ON, L8S 4M1, Canada. \\ ${ }^{3}$ NORDITA, Blegdamsvej-17, Copenhagen-2100, Denmark.
}

\begin{abstract}
We examine the fate of supersymmetric flat directions. We argue that the non-perturbative decay of the flat direction via preheating is an unlikely event. In order to address this issue, first we identify the physical degrees of freedom and their masses in presence of a large flat direction VEV (Vacuum Expectation Value). We explicitly show that the (complex) flat direction and its fermionic partner are the only light physical fields in the spectrum. If the flat direction VEV is much larger than the weak scale, and it has a rotational motion, there will be no resonant particle production at all. The case of multiple flat directions is more involved. We illustrate that in many cases of physical interest, the situation becomes effectively the same as that of a single flat direction, or collection of independent single directions. In such cases preheating is not relevant. In an absence of a fast nonperturbative decay, the flat direction survives long enough to affect thermalization in supersymmetric models as described in hep-ph/0505050 and hep-ph/0512227. It can also "terminate" an early stage of non-perturbative inflaton decay as discussed in hep-ph/0603244.
\end{abstract}

The Minimal Supersymmetric Standard Model (MSSM) has a large number of flat directions, along which the scalar potential vanishes. The flat directions are parameterized by gauge-invariant monomials [1, 2], for a review see [3]. Recently it has been pointed out that these flat directions can play an important role in thermal history of the Universe within supersymmetry (SUSY) [4, 5]. We remind that the thermalization rate depends on $2 \leftrightarrow 2$ and $2 \rightarrow 3$ scatterings of the inflaton decay products. The former lead to kinetic equilibrium but do not change the total number of particles. However, after the last stage of (perturbative) inflaton decay, the reheat plasma is far from chemical equilibrium as the number of particles is much less than that in full thermal equilibrium [4]. As pointed out in [6], $2 \rightarrow 3$ scatterings with gauge boson exchange in the $t$-channel play the main role in establishing chemical equilibrium. For massless gauge bosons, they are infrared divergent. In a non-supersymmetric set up the plasma mass of gauge bosons provides a natural infrared cut-off which regulates these processes(for details see [7]). Since right after the inflaton decay the reheat plasma is dilute, the resulting plasma mass is small. Thus $2 \rightarrow 3$ scatterings are enhanced and additional particles are efficiently produced. In consequence, full thermal equilibrium is established quickly in this case [6].

However the situation will be completely different within supersymmetry because of the flat directions. During inflation these flat directions develop large Vacuum Expectation Value (VEV), see for details [3]. A large VEV induces large SUSY preserving masses to gauge bosons and gauginos via the Higgs mechanism [4] ${ }^{1}$.

\footnotetext{
1 There are ways to suppress the flat direction VEV, if the MSSM flat direction is heavy during inflation then the flat direction settles down to the minimum of its potential. However this happens for a very special case of the Kähler potential, see for details [3]
}

The $2 \rightarrow 3$ scatterings mediated by the gauge bosons are therefore strongly suppressed and, consequently, thermalization will be very slow [4, 5]. We also showed that for a Standard Model (SM) singlet inflaton the inflaton decay is typically perturbative within SUSY [4, 8] ${ }^{2}$.

Delayed thermalization has important outcomes: the Universe undergoes a quasi-thermal phase for a long period after the inflaton has completely decayed, during which the reheat plasma is dilute and far from chemical equilibrium [4, 5]. After the flat direction starts oscillating, its VEV and its induced mass for gauge bosons/gauginos is redshifted by the Hubble expansion. Gradually thermalization rate increases and full thermal equilibrium is established shortly after this rate becomes comparable to the expansion rate of the Universe. A full thermalization typically happens much later than the inflaton decay, leading to a reheat temperature (much) lower than naively expected [4]. The exact value of the reheat temperature depends on the nature of the flat direction and its initial VEV and, typically it is in the range $T_{\mathrm{R}} \sim 10^{4}-10^{7} \mathrm{GeV}$ [4].

The notable off-shoots of a delayed thermalization and a low reheat temperature are ${ }^{3}$ :

- Thermal gravitino production is suppressed. SUSY provides a natural solution to the infamous thermal gravitino problem [4, 5]. Note that the abundance of non-thermal gravitinos is not affected [14, 15],

\footnotetext{
2 In almost all models of inflation, the inflaton is treated as a SM gauge singlet. Very recently we constructed models of inflation which are gauge invariant combinations of squarks and sleptons [9], and with right-handed sneutrinos [10]. The model predictions are trustable and robust (see for other gauge invariant inflatons [11]). We emphasize that this novelty is solely due to the fact that the inflaton is charged under the MSSM gauge group.

3 A late thermalization holds the key to a successful MSSM curvaton mechanism [12], see also [13].
} 
but they do not give rise to a large gravitino abundance any way.

- Thermal leptogenesis within MSSM does not work in most of the parameter space [4, 5].

The above conclusions hold if the flat direction VEV remains large for a sufficiently long time after the onset of its oscillations. This is the case if the flat direction decays perturbatively. Note that the flat direction VEV induces a large mass to all fields which are coupled to it. Perturbative one-particle decay to these fields is only possible when their mass, hence the flat direction VEV, is less than the flat direction mass. If the initial VEV is much larger than the weak scale, this happens much later than the oscillations start.

Recently it has been pointed out in [16] that, the flat direction oscillations can decay very rapidly (i.e., within a few oscillations) via preheating [17, 18]. This will have important implications for cosmology. For example the Universe will quickly thermalize even in the presence of a flat direction with a large VEV. Here we examine this possibility in some detail and argue that the decay of MSSM flat directions via preheating is unlikely in cases of physical interest.

First of all, we identify the physical degrees of freedom and the flat direction masses when a single flat direction has a non-zero VEV. We show that the flat direction and its fermionic partner are the only light fields in the relevant spectrum. As a result, the flat direction motion, so long as it has a rotational component, results in time variation of the mass eigenstates and eigenvalues of superheavy fields. This, however, is not significant at all and does not lead to resonant particle production [20, 21].

Next, we consider the case with more than one flat directions. Two or more flat directions which are simultaneously $D$ - and $F$-flat can develop large VEVs at the same time. Within MSSM the only directions which are completely independent are the two represented by the LLe and $u d d$ monomials. In this case both the flat directions evolve independently and their dynamics is equivalent to a single flat direction, and hence there will be no preheating. We also emphasize that preheating will not be relevant when two flat directions have very different VEVs. This happens in many cases since the directions under consideration are lifted by different $F$-terms which become important at different field values.

The second possibility is multiple flat directions associated with a gauge-invariant polynomial (when all families of leptons and quark are taken into account). This is a new case and very involved, even for a simple example of $H_{u} L$ monomial, whose dynamics was first described in Ref. [22], but preheating effects were not discussed. In a general case, however, the motion of the flat direction $\mathrm{VEV}$ is not known yet. One cannot embark on the issue of particle creation before knowing the details of the flat direction motion for such a scenario. We will briefly sketch this scenario and we think preheating is unimportant for practical purposes. Moreover, due to $F$-term lifting, in many cases the flat manifold has complex dimension one at very large field values. In such cases the situation becomes similar to that of a single flat direction.

The central message is: preheating of an MSSM flat direction is unimportant for physically interesting cases, thus, the flat direction lives long enough to affect reheating and thermalization.

\section{SPONTANEOUS SYMMETRY BREAKING AND PHYSICAL DEGREES OF FREEDOM}

A crucial point is to identify the physical degrees of freedom and their mass spectrum in presence of a nonzero flat direction VEV 4 .

Let us consider the simplest flat direction, which includes only two fields: $H_{u} H_{d}$. This is also familiar from the electroweak symmetry breaking in MSSM. A clear and detailed discussion is given in [23]. Here we briefly recount the situation.

One can always rotate the field configuration to a basis where, up to an overall phase, $H_{u}^{1}=H_{d}^{2}=0$ and $H_{u}^{2}=H_{d}^{1}=\phi_{0} / \sqrt{2}$. Here superscripts denote the weak isospin components of the Higgs doublets. In this basis the complex scalar field is defined by:

$$
\varphi=\frac{\left(H_{u}^{2}+H_{d}^{1}\right)}{\sqrt{2}}
$$

represents a flat direction. Its $\mathrm{VEV}$ breaks the $S U(2)_{W} \times$ $U(1)_{Y}$ down to $U(1)_{\text {em }}$ (in exactly the same fashion as in the electroweak vacuum). The $W^{ \pm}$and $Z$ gauge bosons then obtain masses $m_{W}, m_{Z} \sim g \phi_{0}$ from their couplings to the Higgs fields via covariant derivatives ( $g$ denotes a general gauge coupling).

We also have:

$$
\chi_{1}=\frac{\left(H_{u}^{2}-H_{d}^{1}\right)}{\sqrt{2}}, \text { and } \chi_{2}=\frac{\left(H_{u}^{1}+H_{d}^{2}\right)}{\sqrt{2}} .
$$

Then $\chi_{2}$ and $\chi_{1, R}$ ( $R$ and $I$ denote the real and imaginary parts of a complex scalar field respectively) acquire masses equal to $m_{W}$ and $m_{Z}$, respectively, through the $D$-term part of the scalar potential. Note that

$$
\chi_{3}=\frac{\left(H_{u}^{1}-H_{d}^{2}\right)}{\sqrt{2}},
$$

and $\chi_{1, I}$ are the three Goldstone bosons, which are eaten by the gauge fields via the Higgs mechanism. Therefore, out of 8 real degrees of freedom in the two Higgs doublets, there are only two light physical fields: $\varphi_{R}, \varphi_{I}$. They are

\footnotetext{
4 The first version of Ref. [16] did not count correctly the light degrees of freedom. Then it was claimed that even a single MSSM flat direction can decay non-perturbatively. However, as we argue below, this is not the case.
} 
exactly massless when SUSY is not broken (and there is no $\mu$ term either).

An important point is that the masses induced by the flat direction VEV are SUSY conserving. One therefore finds the same mass spectrum in the fermionic sector. More specifically, the Higgsino fields $\widetilde{H}_{u}^{1}$ and $\widetilde{H}_{d}^{2}$ are paired with the Winos, while $\left(\widetilde{H}_{u}^{2}-\widetilde{H}_{d}^{1}\right) / \sqrt{2}$ is paired with the Zino to acquire masses equal to $m_{W}$ and $m_{Z}$, respectively, through the gaugino-gauge-Higgsino interaction terms. The fermionic partner of the flat direction $\left(\widetilde{H}_{u}^{2}+\widetilde{H}_{d}^{1}\right) / \sqrt{2}$ remains massless (note that the photon and photino are also massless, but not relevant for our discussion).

In reality, supersymmetry is broken and, $\varphi$ obtains a mass $m_{\varphi} \sim \mathcal{O}(\mathrm{TeV})$ from soft SUSY breaking term (the same is true for the gauginos). However, for $g \varphi_{0} \gg$ $\mathcal{O}(\mathrm{TeV})$, which is the situation relevant to the early Universe, the mass spectrum is hierarchical: $\chi_{1}, R, \chi_{2}$, and gauge fields (plus their fermionic partners) are superheavy.

In a general case the total number of light scalars, $N_{\text {light }}$, is given by:

$$
N_{\text {light }}=N_{\text {total }}-\left(2 \times N_{\text {broken }}\right),
$$

where $N_{\text {total }}$ is the total number of scalar degrees of freedom, and $N_{\text {broken }}$ is the number of spontaneously broken symmetries. Note that the factor 2 counts for the number of eaten Goldstone bosons plus the number of degrees of freedom which have obtained large masses equal to those of the gauge bosons. In the case of $H_{u} H_{d}$ direction, Eq. (4) reads: $2=8-(2 \times 3)$.

\section{PARTICLE PRODUCTION FROM A SINGLE FLAT DIRECTION}

A generic MSSM flat direction has $F$-term and $D$ term couplings to other fields. The dynamics and nonperturbative particle creation for a flat direction with $F$ term couplings are unimportant. This has been clearly illustrated in Refs. 21, 24], here we will not discuss this case. We will only concentrate on the $D$-terms.

For the $H_{u} H_{d}$ flat direction, supersymmetric $D$-term contribution lead to:

$$
\begin{gathered}
V_{D}=\left(a_{1} g_{1}^{2}+a_{2} g_{2}^{2}\right)\left[\varphi_{R} \chi_{1, R}+\varphi_{I} \chi_{1, I}\right]^{2}+ \\
a_{2} g_{2}^{2}\left[\varphi_{R} \chi_{2, R}+\varphi_{I} \chi_{2, I}\right]^{2}+a_{2} g_{2}^{2}\left[\varphi_{R} \chi_{3, R}+\varphi_{I} \chi_{3, I}\right]^{2}
\end{gathered}
$$

with $a_{1}, a_{2} \sim \mathcal{O}\left(10^{-1}\right)$. Here $g_{1}$ and $g_{2}$ are gauge couplings of $U(1)_{Y}$ and $S U(2)_{W}$. For a general monomial the $D$-term contribution will be similar when the scalars are decomposed to the flat direction $\varphi$ and orthogonal fields $\chi$.

Let us now consider the case where the flat direction VEV is oscillating. The oscillations start when the Hubble expansion rate drops below the flat direction mass, i.e., when $H(t) \simeq m_{\varphi}$. Note that the motion is due to soft supersymmetry breaking terms and the $F$-terms [1] as $V_{D}$ identically vanishes for a flat direction. The soft mass and superpotential contribution depends on the modulus of flat direction $|\varphi|$. On the other hand, the " $A^{\prime \prime}$-terms result in a phase-dependent contribution, which exerts a torque and generates a rotation in the $\varphi$ plane. As a generic feature the flat direction motion has a major rotational component [1].

\section{A. Rotation on a circle}

If the real and imaginary parts of the flat direction oscillate with the same amplitude, $\varphi_{0}$, but with a $\pi / 2$ phase difference, the trajectory will be a circle in the $\varphi$ plane: $\varphi=\varphi_{0} \exp \left(i m_{\varphi} t\right)$. This is not what exactly happens in the early Universe, but as we just pointed out, this is a very good approximation of a generic situation.

The first term on the right-hand side of Eq. (5), which arises from the diagonal part of $S U(2)_{W}$ and $U(1)_{Y}$, gives rise to ${ }^{5}$ :

$$
V \sim\left(g_{1}^{2}+g_{2}^{2}\right) \varphi_{0}^{2}\left[\cos \left(m_{\varphi} t\right) \chi_{1, R}+\sin \left(m_{\varphi} t\right) \chi_{1, I}\right]^{2} .
$$

At a first glance it seems that there are two scalar mass eigenstates:

$$
\begin{aligned}
\vec{\omega}_{1}(t) & =\cos \left(m_{\varphi} t\right) \chi_{1, R}+\sin \left(m_{\varphi} t\right) \chi_{1, I}, \\
\vec{\omega}_{2}(t) & =\cos \left(m_{\varphi} t\right) \chi_{1, I}-\sin \left(m_{\varphi} t\right) \chi_{1, R},
\end{aligned}
$$

where $\omega_{1}$ has a mass $\sim g \varphi_{0}$, hence superheavy, and $\omega_{2}$ is light. Note that the mass eigenvalues are constant in time (despite rotation of $\varphi$ ) but the mass eigenstates are evolving.

In general one can picture an instantaneous mass eigenstate by a vector, $\vec{\omega}(t)$, whose magnitude represents the corresponding mass eigenvalue. In the case at hand the mass eigenstates are two-dimensional vectors in the $\chi_{1}$ plane. One can then expect non-perturbative production of particles whenever the adiabaticity is violated in the evolution of $\vec{\omega}$, i.e., such that

$$
\left|\frac{d \vec{\omega}(t)}{d t}\right| \gtrsim|\vec{\omega}(t)|^{2}
$$

It can be shown from Eq. (7) that this is the case at all moments of time for the light mass eigenstate $\vec{\omega}_{2}(t)$. One might then conclude that quanta of the light mode are copiously produced via parametric resonance, thus, leading to a quick decay of the flat direction oscillations [16].

\footnotetext{
${ }^{5}$ Contributions from non-diagonal parts of $S U(2)_{W}$ lead to a similar contribution with $\chi_{1}$ being replaced by $\chi_{2}, \chi_{3}$.
} 
However, a closer inspection shows that $\vec{\omega}_{2}(t)$ actually is one of the three Goldstone bosons in the background of rotating $\varphi$, see Eq. (2), and the subsequent discussions.

Hence $\vec{\omega}_{2}(t)$ is not a physical degree of freedom as it is eaten by the $Z$ gauge boson. Indeed, as we elaborated, there are no physical light fields except the rotating flat direction. Obviously the mass eigenstates and eigenvalues of the flat direction are not affected by its rotation and therefore they are constant in time, see Eq. (1).

Further note that the rotation only results in a time variation in the mass eigenstates of the heavy fields, and the corresponding eigenvalues (which are constant in time) are much larger than the frequency of rotation.

At the onset of the flat direction oscillations, we typically have, $g \varphi_{0} \geq 10^{8} m_{\varphi}$. This implies that the adiabaticity condition always holds, see Eq. (8), and hence there will be no resonant particle production ${ }^{6}$.

In an absence of particle production, the flat direction VEV is only subject to redshifting by the Hubble expansion. In a Universe dominated by relativistic particles, not necessarily thermalized, we have $\langle\varphi\rangle \propto H^{3 / 4}$. Therefore, the heavy fields become continuously lighter.

The resonant particle production, due to timevariation of the mass eigenstates, if at all becomes important, will only be relevant when $g\langle\varphi\rangle \sim m_{\varphi}$. However, this will happen much later than the onset of the flat direction oscillations, indeed, we find:

$$
H_{\text {preheat }} \sim\left(\frac{m_{\varphi}}{g \varphi_{0}}\right)^{4 / 3} m_{\varphi} \leq 10^{-9} m_{\varphi}
$$

where $m_{\varphi} \sim \mathcal{O}(\mathrm{TeV})$. This implies that the flat direction VEV survives a very long time, which is sufficient to affect thermalization along the lines pursued in Refs. [4, 5]. In particular, Eq. (9) can be translated into an upper bound on the reheat temperature: $T_{\mathrm{R}} \sim\left(H_{\text {preheat }} M_{\mathrm{P}}\right)^{1 / 2} \leq 10^{6} \mathrm{GeV}$. This is compatible even with the most stringent limit on the reheat temperature, which is obtained for unstable gravitinos with a dominant hadronic decay mode [19].

\section{B. A General Rotation}

For a general rotation we will have: $\varphi_{R}=\varphi_{0} \cos \left(m_{\varphi} t\right)$ and $\varphi_{I}=a \varphi_{0} \cos \left(m_{\varphi} t+\theta\right)$, where $a \leq 1$ is a positive number and $\theta$ is an $\mathcal{O}(1)$ phase.

In this case, both the mass eigenstates and mass eigenvalues of the heavy fields change in time. The mass eigenvalues, given by $\sim g|\varphi|$, lie in the range $\sim\left[a g \varphi_{0}, g \varphi_{0}\right]$.

\footnotetext{
${ }^{6}$ Note that for a circular rotation, the Lagrangian for the heavy fields is the same as that in a static case up to corrections of order $\left(m_{\varphi} / g \varphi_{0}\right)$, coming form time-derivatives of $\varphi$. Therefore, one can reliably use the same mass eigenstates and eigenvalues as that of the static case.
}

Particle production will be insignificant so long as the time evolution of the system is adiabatic. The adiabaticity condition for mass eigenvalues is violated if:

$$
g \frac{d|\varphi|}{d t} \gtrsim g^{2}|\varphi|^{2}
$$

This happens when $g|\varphi| \lesssim a\left(g \varphi_{0} m_{\varphi}\right)^{1 / 2}$, and provided that $a<\left(m_{\varphi} / g \varphi_{0}\right)^{1 / 2}[20,21]$. For a typical initial condition, where $g \varphi_{0} \geq 10^{8} m_{\varphi}$, this requires that, $a \leq 10^{-4}$. As mentioned earlier, in a generic situation we have $a \sim \mathcal{O}(1)[1]$.

For such small values of " $a^{\prime \prime}$, the flat direction motion will be effectively one-dimensional: $\varphi_{R} \approx \varphi_{0} \cos \left(m_{\varphi} t\right)$ and $\varphi_{I} \approx 0$. In this case, $D$-terms result in an interaction term $g^{2} \varphi_{R}^{2} \chi_{R}^{2}$, see Eq. (6), which leads to a fast decay of flat direction oscillations via the standard picture of preheating [18]. However, the challenge is to seek, $a \leq$ $10^{-4}$, in a physical models.

We conclude that in general; the flat direction oscillations survive a very long time. A fast decay can only happen in the exceptional case when the flat direction motion is effectively one-dimensional.

\section{TWO OR MORE FLAT DIRECTIONS}

A necessary (but not sufficient) condition for a quick decay of an MSSM flat direction via preheating is that; there must exist light scalar degrees of freedom whose mass eigenstates and/or eigenvalues are evolving in time in a non-adiabatic fashion. As we showed this does not happen for a single flat direction. Here we examine the situation where there are multiple flat directions. We discuss two possible cases separately.

\section{A. Two directions represented by different monomials}

Two directions, which are simultaneously $D$ - and $F$ flat, can independently acquire large VEVs during inflation. An an important example, there exists two flat directions represented by the $u d d$ and $L L e$ monomials, respectively. Note that $u$ and $d$ have no Yukawa couplings to $L$ and $e$. This guarantees that they are simultaneously $F$-flat. The $D$-flatness imposes that the two $d$ 's in $u d d$, and the two $L$ 's in $L L e$ are from different families. Also $F$-flatness requires that $e$ belong to a third lepton family. Without any loss of generality, let us consider two flat directions, parameterized as:

$$
\begin{aligned}
& u_{1}^{1}=d_{1}^{2}=d_{2}^{3}=\frac{\varphi_{0}}{\sqrt{3}}, \quad(\text { up to a phase }) \\
& \left.L_{1}^{1}=L_{2}^{2}=e_{3}=\frac{\varphi_{0}^{\prime}}{\sqrt{3}}, \quad \text { (up to a phase }\right)
\end{aligned}
$$

Here the upper indices indicate color (for $u$ and $d$ ) and weak isospin (for $L$ ). The lower indices denote the family. 
Note that $u$ can be from any of the quark families, and we have chosen, 1 , just as one possible case. Then the flat directions $\varphi$ and $\varphi^{\prime}$ are given by:

$$
\varphi=\frac{\left(u_{1}^{1}+d_{1}^{2}+d_{2}^{3}\right)}{\sqrt{3}}, \quad \varphi^{\prime}=\frac{\left(L_{1}^{1}+L_{2}^{2}+e_{3}\right)}{\sqrt{3}}
$$

with respective masses:

$m_{\varphi}^{2}=\frac{\left(m_{u_{1}}^{2}+m_{d_{1}}^{2}+m_{d_{2}}^{2}\right)}{3}, m_{\varphi^{\prime}}^{2}=\frac{\left(m_{L_{1}}^{2}+m_{L_{2}}^{2}+m_{e_{3}}^{2}\right)}{3}$.

Note that $\varphi$ and $\varphi^{\prime}$ together break all of the SM gauge symmetries, hence, they can affect thermalization of inflaton decay products [4].

These two directions independently oscillate with amplitudes $\varphi_{0}$ and $\varphi_{0}^{\prime}$, respectively, and frequencies $m_{\varphi}$ and $m_{\varphi^{\prime}}$ respectively.

There are four light scalar degrees of freedom which correspond to $\varphi$ and $\varphi^{\prime}$. The total number of scalars is $N_{\text {total }}=28$, with 18 contained in the $u_{1} d_{1} d_{2}$ monomial and 10 in the $L_{1} L_{2} e_{3}$ monomial. Since the SM gauge group is completely broken, we have $N_{\text {broken }}=12$. Then Eq. (4) results in $N_{\text {light }}=28-24=4$.

This implies that $\varphi$ and $\varphi^{\prime}$ are the only light fields around. Their mass eigenstates and eigenvalues, given by Eqs. (12) and (13), respectively, are constant in time and therefore not affected by their oscillations. Therefore, the condition in Eq. (8) will not be satisfied. There are time variations in the mass eigenstates and eigenvalues of the heavy fields. However, as discussed in the previous section, so long as $\varphi$ and $\varphi^{\prime}$ have rotational motion, resonant particle production will be negligible [20, 21].

There are many examples where the fields in the two monomials can have Yukawa couplings to each other. In these cases simultaneous $F$-flatness of the two directions is not guaranteed in general. For an example, let us consider the two directions represented by $Q Q Q L$ and $L L d d d$ monomials. Note that $Q$ and $d$ can be coupled to each other through quark Yukawas. If this is the case, then the two directions cannot develop large VEVs simultaneously.

However, Yukawas couple fields with the same color index to each other. One can then choose color indices such that $Q$ and $d$ from the same family have different colors. This implies that there is a subset of directions which are simultaneously $D$ - and $F$-flat, and hence, can develop large VEVs independently. The situation will be similar for the directions represented by $u d d$ and $Q L d$ monomials ${ }^{7}$.

\footnotetext{
7 The second version of Ref. [16] also cites other examples of two independent flat directions such as $L L e$ and $Q L d$. However, simultaneous $F$-flatness of these two directions requires that the $L$ in $Q L d$ must belong to the same family as one of the two $L$ 's in $L L e$. Therefore one cannot have two independent directions parameterized as $L=L=e=\varphi_{0}$ and $Q=L=d=\varphi_{0}^{\prime}$ (up to two independent phases). It is possible that $Q, L, d$, and $e$ all
}

In the above mentioned cases, one finds $N_{\text {light }}>4$, which implies that there are light degrees of freedom besides the two oscillating flat directions. This however is not a sufficient condition to have preheating. To elucidate this point, let us consider a toy model with a $U(1)$ gauge group and four scalar fields $\phi_{1}, \phi_{1}^{\prime}, \phi_{2}, \phi_{2}^{\prime}$ with respective charges $+1,+1,-1,-1$. The corresponding $D$ term is

$$
V_{D} \sim g^{2}\left(\left|\phi_{1}\right|^{2}-\left|\phi_{2}\right|^{2}+\left|\phi_{1}^{\prime}\right|^{2}-\left|\phi_{2}^{\prime}\right|^{2}\right)^{2}
$$

After the following field redefinition

$$
\begin{aligned}
& \varphi=\frac{\left(\phi_{1}+\phi_{2}\right)}{\sqrt{2}}, \varphi^{\prime}=\frac{\left(\phi_{1}^{\prime}+\phi_{2}^{\prime}\right)}{\sqrt{2}} \\
& \chi=\frac{\left(\phi_{1}-\phi_{2}\right)}{\sqrt{2}}, \chi^{\prime}=\frac{\left(\phi_{1}^{\prime}-\phi_{2}^{\prime}\right)}{\sqrt{2}}
\end{aligned}
$$

we have ${ }^{8}$

$$
V_{D} \sim g^{2}\left(\varphi_{R}^{\prime} \chi_{R}^{\prime}+\varphi_{I}^{\prime} \chi_{I}^{\prime}+\varphi_{R} \chi_{R}+\varphi_{I} \chi_{I}\right)^{2} .
$$

where $R$ and $I$ denote the real and imaginary components of a scalar field respectively. We consider flat directions with maximal rotation ${ }^{9}$ :

$$
\begin{aligned}
\varphi_{R}=\varphi_{0} \cos \left(m_{\varphi} t+\theta\right) & , \varphi_{I}=\varphi_{0} \sin \left(m_{\varphi} t+\theta\right) \\
\varphi_{R}^{\prime}=\varphi_{0}^{\prime} \cos \left(m_{\varphi^{\prime}} t\right) & , \varphi_{I}^{\prime}=\varphi_{0}^{\prime} \sin \left(m_{\varphi^{\prime}} t\right),
\end{aligned}
$$

where $\theta$ is a constant phase. There is a total of 8 scalar degrees of freedom. Four of them $\varphi_{R}, \varphi_{I}, \varphi_{R}^{\prime}, \varphi_{I}^{\prime}$ represent the two complex flat directions. The mass matrix for the other four degrees of freedom can be diagonalized to obtain instantaneous mass eigenstates. It is readily seen from Eq. (16) that one degree of freedom

$$
\begin{aligned}
\chi_{1}(t) & =\left(\varphi_{0}^{2}+\varphi_{0}^{\prime 2}\right)^{-1 / 2}\left[\varphi_{0}^{\prime} \cos \left(m_{\varphi^{\prime}} t\right) \chi_{R}^{\prime}+\varphi_{0}^{\prime} \sin \left(m_{\varphi^{\prime}} t\right) \chi_{I}^{\prime}\right. \\
& \left.+\varphi_{0} \cos \left(m_{\varphi} t+\theta\right) \chi_{R}+\varphi_{0} \sin \left(m_{\varphi} t+\theta\right) \chi_{I}\right]
\end{aligned}
$$

has a mass $\sim g\left(\varphi_{0}^{2}+\varphi_{0}^{\prime 2}\right)^{1 / 2}$ and is superheavy. Spontaneous breakdown of the $U(1)$ by the VEV of two flat directions yields a Goldstone boson

$$
\begin{aligned}
\chi_{2}(t) & =\left(\varphi_{0}^{2}+\varphi_{0}^{\prime 2}\right)^{-1 / 2}\left[-\varphi_{0}^{\prime} \sin \left(m_{\varphi^{\prime}} t\right) \chi_{R}^{\prime}+\varphi_{0}^{\prime} \cos \left(m_{\varphi^{\prime}} t\right) \chi_{I}^{\prime}\right. \\
& \left.-\varphi_{0} \sin \left(m_{\varphi} t+\theta\right) \chi_{R}+\varphi_{0} \cos \left(m_{\varphi} t+\theta\right) \chi_{I}\right]
\end{aligned}
$$

have a non-zero VEV, but the situation will actually be similar to that of multiple flat directions, which will be discussed later. This is also the case for two directions represented by $L L e$ and LLddd monomials.

${ }^{8}$ A general $D$-term has the same structure, see Eq. (5). It is therefore straightforward to generalize this discussion made for a $U(1)$ gauge group.

${ }^{9}$ Generalization to the case with general rotation is straightforward. 
which is eaten up via the Higgs mechanism (hence removed from the spectrum). Note that in the special cases where $\varphi_{0}=0$ or $\varphi_{0}^{\prime}=0$, the expression in Eq. (19) is reduced to that for a single flat direction, given in the second line of Eq. (7).

Finally, there are two physical light degrees of freedom

$$
\chi_{3}(t)=\left(\varphi_{0}^{2}+\varphi_{0}^{\prime 2}\right)^{-1}\left(a_{1} \chi_{R}^{\prime}+a_{2} \chi_{I}^{\prime}+a_{3} \chi_{R}+a_{4} \chi_{I}\right)
$$

and

$$
\chi_{4}(t)=\left(\varphi_{0}^{2}+\varphi_{0}^{\prime 2}\right)^{-1}\left(b_{1} \chi_{R}^{\prime}+b_{2} \chi_{I}^{\prime}+b_{3} \chi_{R}+b_{4} \chi_{4}\right)
$$

where

$$
\begin{aligned}
& a_{1}=-\varphi_{0}^{2}+\varphi_{0} \varphi_{0}^{\prime} \cos (\Delta m t+\theta) \\
& a_{2}=-\varphi_{0} \varphi_{0}^{\prime} \sin (\Delta m t+\theta) \\
& a_{3}=-\varphi_{0}^{\prime 2}+\varphi_{0} \varphi_{0}^{\prime} \cos (\Delta m t+\theta) \\
& a_{4}=\varphi_{0} \varphi_{0}^{\prime} \sin (\Delta m t+\theta),
\end{aligned}
$$

and

$$
\begin{aligned}
& b_{1}=-\varphi_{0} \varphi_{0}^{\prime} \sin (\Delta m t+\theta), \\
& b_{2}=\varphi_{0}^{2}-\varphi_{0} \varphi_{0}^{\prime} \cos (\Delta m t+\theta) \\
& b_{3}=\varphi_{0} \varphi_{0}^{\prime} \sin (\Delta m t+\theta) \\
& b_{4}=\varphi_{0}^{\prime 2}-\varphi_{0} \varphi_{0}^{\prime} \cos (\Delta m t+\theta) .
\end{aligned}
$$

These two eigenstates have a soft supersymmetry breaking mass $m_{\chi} \sim \mathcal{O}(\mathrm{TeV})$. Here $\Delta m \equiv m_{\varphi}-m_{\varphi^{\prime}}$ is the mass difference of the two flat directions.

Time variation in $\chi_{3}(t), \chi_{4}(t)$ may lead to resonant particle production. Note however that this can happen only if $m_{\varphi} \neq m_{\varphi^{\prime}}$. Indeed it can be seen from Eqs. (20|21) that there will be no time-dependent terms in $\chi_{3}(t), \chi_{4}(t)$ if the flat directions have the same mass. This can be qualitatively understood as following. The two rotating flat directions can be visualized as two vectors which rotate in the field space. So long as the flat directions have the same mass, the two vectors rotate with the same frequency, and so does the resultant vector from their addition. Therefore the situation is effectively reduced to that of one rotating flat direction. In this case, as explained in the previous section, all time variation is in the sector of heavy fields. Neither the mass eigenstates nor the mass eigenvalues of the physical light fields are time-dependent. In consequence, there will be no resonant particle production.

Having $\Delta m \neq 0$ is necessary but not sufficient for significant particle production. To demonstrate this let us consider two flat directions with hierarchically different VEVs. Without loss of generality, we assume that $\varphi_{0}^{\prime} \ll \varphi_{0}$. Then, to the leading order in $\varphi_{0}^{\prime} / \varphi_{0}$, we have

$$
\begin{gathered}
\chi_{3}(t)=-\chi_{R}^{\prime}+\left(\frac{\varphi_{0}^{\prime}}{\varphi_{0}}\right) \\
{\left[\cos (\Delta m t+\theta)\left(\chi_{R}^{\prime}+\chi_{R}\right)+\sin (\Delta m t+\theta)\left(-\chi_{I}^{\prime}+\chi_{I}\right)\right],}
\end{gathered}
$$

and

$$
\begin{gathered}
\chi_{4}(t)=\chi_{I}^{\prime}+\left(\frac{\varphi_{0}^{\prime}}{\varphi_{0}}\right) \\
{\left[\sin (\Delta m t+\theta)\left(-\chi_{R}^{\prime}+\chi_{R}\right)-\cos (\Delta m t+\theta)\left(\chi_{I}^{\prime}+\chi_{I}\right)\right] .}
\end{gathered}
$$

The important point is that $\varphi_{0}^{\prime} / \varphi_{0}$ appears in front of the time-dependent terms in Eqs. (2425) ${ }^{10}$. Temporal derivatives of these terms which show up in the equation of motion of $\chi_{3}(t), \chi_{4}(t)$ modes will therefore be suppressed by this factor. It follows from the analysis of [16] that modes with a physical momentum $k \leq \Delta k$ will undergo resonance where

$$
\Delta k \simeq \sqrt{\left(\frac{\varphi_{0}^{\prime}}{\varphi_{0}}\right)\left(m_{\varphi}-m_{\varphi^{\prime}}\right)^{2}-m_{\chi}^{2}} .
$$

Had $\chi_{3}(t), \chi_{4}(t)$ been massless (i.e. $m_{\chi}=0$ ), the width of the resonance band would have shrunk $\propto\left(\varphi_{0}^{\prime} / \varphi_{0}\right)^{1 / 2}$. Modes within the resonance band were exponentially amplified, though the required time scale would increase by a $\operatorname{logarithmic}$ factor $\log \left(\varphi_{0} / \varphi_{0}^{\prime}\right)$ due to phase space suppression. However supersymmetry breaking inevitably results in a soft mass $m_{\chi} \sim \mathcal{O}(\mathrm{TeV})$. Hence the resonance band completely disappears when ${ }^{11}$

$$
\frac{\varphi_{0}}{\varphi_{0}^{\prime}} \gtrsim\left(\frac{m_{\varphi}-m_{\varphi^{\prime}}}{m_{\chi}}\right)^{2} .
$$

Since $m_{\chi}, m_{\varphi}, m_{\varphi^{\prime}}$ are all set by supersymmetry breaking, even a small hierarchy between $\varphi_{0}^{\prime}$ and $\varphi_{0}$ will be sufficient to kill off the resonance band. Typically there will be no non-perturbative particle production if $\varphi_{0} \geq 10 \varphi_{0}^{\prime} 12$.

We remind that the VEV of a flat direction at the onset of its oscillations is set by the higher-order superpotential term of the form $\varphi^{n} / M^{n-3}$ (induced by physics at a high scale $M$ ) which lifts it: $\varphi_{0} \sim\left(m_{\varphi} M^{n-3}\right)^{1 / n-2}$ [1]. Directions which are represented by different monomials usually are lifted by terms with different values of $n$, and hence have different VEVs. For example, $Q L d$ and $Q Q Q L$ are lifted by $n=4$ terms, while $u d d$ and $L L d d d$ survives until $n=6$ and $n=7$ respectively [2]. Consider any two such flat directions $\varphi^{\prime}$ and $\varphi$ which are lifted by superpotential terms of order $m$ and $n$ respectively $(3<m<n)$. For $M=M_{\mathrm{P}}$ or $M=M_{\mathrm{GUT}}$, we indeed

10 In the opposite case when $\varphi_{0} \ll \varphi_{0}^{\prime}$, the expressions for $\chi_{3}(t)$ and $\chi_{4}(t)$ are obtained by changing $\varphi \leftrightarrow \varphi^{\prime}, \chi_{R, I} \leftrightarrow \chi_{R, I}^{\prime},(\Delta m t+$ $\theta) \rightarrow-(\Delta m t+\theta)$ in Eqs. (24/25). The time-dependent terms are in this case suppressed by $\varphi_{0} / \varphi_{0}^{\prime} \ll 1$.

11 This point is ignored in the third version of [16], see its added note.

12 Note that Hubble expansion redshifts the VEV of two flat directions the same. Hence Eq. (26) determines the width of resonance band throughout the expansion of Universe. 
have $\varphi_{0} \geq 10 \varphi_{0}^{\prime}$ for all cases with $m \neq n$. Then, as we explained above, there will be no preheating at all.

One comment is in order before closing this subsection. As we discussed in section 1, the masses induced by the VEV of flat directions are supersymmetry conserving. This implies that the spectrum of fermions is the same as that of bosons except for the soft supersymmetry breaking effects. In particular, the eigenstates of fermionic partners of $\chi_{3}(t)$ and $\chi_{4}(t)$, denoted by $\tilde{\chi}_{3}(t)$ and $\tilde{\chi}_{4}(t)$ respectively, have the same time dependence as in Eqs.(24|25) ${ }^{13}$. Hence, similar to Eq. (26), corresponding modes with a physical momentum $k \leq \Delta k_{\text {ferm }}$ have resonance instability where

$$
\Delta k_{f e r m} \simeq\left(\frac{\varphi_{0}^{\prime}}{\varphi_{0}}\right)^{1 / 2}\left|m_{\varphi}-m_{\varphi^{\prime}}\right| .
$$

The important difference is that fermionic modes have no supersymmetry breaking mass $m_{\chi}$. Thus the resonance band shrinks as a result of a hierarchy $\varphi_{0}^{\prime} \ll \varphi_{0}$ but, unlike the bosonic case, does not completely disappear. Therefore one might think that flat directions will quickly decay via non-perturbative production of fermions.

Let us estimate the rate for production of fermions The key point is that, due to Pauli blocking, the occupation number of fermions cannot exceed 1. An occupation number $\sim 1$ is achieved within a time $\delta t \sim$ $\Delta k_{\text {ferm }}^{-1}$ [16]. The energy density in the produced particles is $\rho_{\text {ferm }} \sim \Delta k_{\text {ferm }}^{4}$. Hubble expansion and rescatterings deplete the resonance band, after which production of fermions within the band will resume. Assuming that this happens immediately, an amount $\Delta k_{\text {ferm }}^{4}$ of energy density is transferred to fermions within each time interval $\sim \Delta k_{\text {ferm }}^{-1}$. Then the energy density in fermions increases by $\sim\left(\Delta k_{\text {ferm }}^{5} / H\right)$ in one Hubble time. Particle production becomes efficient when this is comparable to the energy density in flat directions ${ }^{14}$. In a Universe dominated by relativistic particles (from inflaton decay), the latter changes $\propto H^{3 / 2}$. With the help of Eq. (28), it turns out that production of fermions will be efficient when $H \ll m_{\varphi}\left(m_{\varphi} / \varphi_{0}\right)^{4 / 5}$. This is sufficiently late in order for the flat directions to affect thermal history of the Universe [4].

\section{B. Multiple directions represented by a polynomial}

When the family indices of lepton and quark multiplets are taken into account, we will have a gauge-invariant polynomial which represent multiple flat directions ${ }^{15}$.

13 Note that we are only interested in the light fields. Hence we do not consider gauge and gaugino fields as they have a mass $\sim g \varphi_{0}$ and are superheavy.

${ }^{14}$ For hierarchical VEVs, i.e. $\varphi_{0} \gg \varphi_{0}^{\prime}$, the energy density is mainly in $\varphi$.

15 In this sense the $H_{u} H_{d}$ monomial is very special, since it is the only one which has no family indices.
For example, let us consider the $H_{u} L_{i}$ polynomial, where $1 \leq i \leq 3$ (for a detailed discussion, see [22]).

Assuming $D$-flatness, one can always go to a basis where $H_{u}^{1}=L_{1}^{2}=L_{2}^{2}=L_{3}^{2}=0$ (superscripts denote weak isospin components), and (up to an overall phase)

$$
\begin{gathered}
H_{u}^{2}=\frac{\varphi_{0}}{\sqrt{2}} \\
L_{1}^{1}=\frac{a \varphi_{0}}{\sqrt{2}}, L_{2}^{1}=\frac{b \varphi_{0}}{\sqrt{2}}, L_{3}^{1}=\frac{\sqrt{1-|a|^{2}-|b|^{2}} \varphi_{0}}{\sqrt{2}},
\end{gathered}
$$

where $|a|^{2}+|b|^{2} \leq 1$.

A flat direction is then given by

$$
\varphi=\frac{\left(H_{u}^{2}+a^{*} L_{1}^{1}+b^{*} L_{2}^{1}+\sqrt{1-|a|^{2}-|b|^{2}} L_{3}^{1}\right)}{\sqrt{2}},
$$

and its mass is:

$$
m_{\varphi}^{2}=\frac{m_{H_{u}}^{2}+|a|^{2} m_{L_{1}}^{2}+|b|^{2} m_{L_{2}}^{2}+\left(1-|a|^{2}-|b|^{2}\right) m_{L_{3}}^{2}}{2} .
$$

Now the flat manifold has complex dimension 3. Note that $N_{\text {total }}=16$ and $N_{\text {broken }}=3$, and hence Eq. (4) results in $N_{\text {light }}=10$. This implies that there are four light degrees of freedom besides those which parameterize the flat manifold.

The trajectory of the flat direction VEV is determined by its initial value, the soft breaking terms and higher order superpotential terms which lift the flatness. The flat direction motion is confined to a complex plane when $a, b$ are constant in time (in particular for $a=b=0, a=0$ and $|b|=1$, or $|a|=1$ and $b=0)$. However, for a general motion on the flat manifold, $a$ and $b$ can assume any values in the allowed ranges, and hence are time dependent. The phase motion of the fields was already recognized in Ref. [22].

However, note that just time variation of mass eigenstates is not a sufficient condition for a quick nonperturbative decay of the flat direction. A closer analysis suggests that the manifold spanning the flat direction is curved and the kinetic terms are non-minimal 22]. It was already noticed in Ref. [22], that a small perturbation of the flat direction trajectory will be highly non-trivial, the trajectories have instability which leads to possibly a chaotic motion. Particle production due to a chaotic motion is much more involved than that for an oscillatory motion and, a detailed investigation is needed to clarify this situation.

Further note, that the other 4 light scalars (besides 6 , which parameterize the flat manifold) are expected to have (self-)interactions with gauge strengths (from the $D$-terms), as they do not correspond to genuinely flat directions, see Eq. (29]30). Such interactions can strongly suppress non-perturbative particle production [24, 25].

An important point is to note that the flat manifold does not remain the same for all field values. As mentioned, higher-order superpotential terms of the form, $\varphi^{n} / M^{n-3}$ (with $n>3$ ), lift the flatness for large field 
values. If one writes all higher order terms which respect the SM gauge symmetries, then none of the MSSM flat directions survive beyond $n=9$ [2]. A higher order term $\varphi^{n} / M^{n-3}$ becomes important at a field value $|\varphi| \sim\left(m_{\varphi} M^{n-3}\right)^{1 / n-2}[1$, 2]. Therefore, terms with different $n$ take action at different VEVs and lifting of the flatness happens at stages. In some physically interesting cases the flat manifold has complex dimension "one" at very large field values, which effectively leaves us with a single flat direction. This happens for the $L L e, L L d d d$ and $Q u Q u e$ polynomials (when all families are taken into account). The flat manifolds corresponding to these polynomials have complex dimension 3,3 , and 16 respectively. However beyond $n=4$, equivalently at field values larger than $\left(m_{\varphi} M\right)^{1 / 2}$, they all become effectively of complex dimension one [2]. These remaining single flat directions are eventually lifted with $n=6, n=7$, and $n=9$ terms respectively [2]. Then, starting at large VEVs, the situation will be similar to that for a single flat direction discussed in the previous section, and hence there will be no preheating.

Finally, as a side comment, we emphasize that the flat directions can "terminate" an early stage of nonperturbative inflaton decay via preheating, regardless of their fate [8]. They can induce large masses to the inflaton decay products at time scales long before the flat direction oscillations start. Hence, contrary to the standard lore [18], the inflaton can decay perturbatively even if it has large couplings to the matter fields.

Within MSSM the only renormalizable interactions of a gauge singlet inflaton, $\Phi$, with matter can happen through superpotential terms of the form $h \Phi H_{u} L$ or $h \Phi H_{u} H_{d}$ [8]. Rest of the superpotential couplings to matter fields are through non-renormalizable terms. The couplings of a singlet to the matter fields through derivative terms (dictated by Kähler potential) will also be non-renormalizable.

In models of large field inflation, a typical VEV of the inflaton is $\langle\Phi\rangle \sim \mathcal{O}\left(M_{\mathrm{P}}\right)$, during and just after inflation. Then any renormalizable couplings of the infla- ton induces a large SUSY conserving mass, $h M_{\mathrm{P}}$, for $H_{u}$ and $L$, or $H_{u}$ and $H_{d}$ multiplets. This implies that neither $H_{u} L$ nor $H_{u} H_{d}$ directions can develop a large VEV during inflation. Hence neither of them are relevant in the process of thermalization, unless the inflaton is very weakly (i.e. gravitationally) coupled to all fields.

\section{CONCLUSIONS}

To conclude, the particle production via preheating does not happen for a single flat direction, it is also not likely for multiple flat directions. In many cases which are of physical interest, after taking into account of the $F$-term constraints and $F$-term lifting, the multiple flat direction case becomes effectively the same as that of a single flat direction, or collection of independent single flat directions. In either cases preheating is not relevant. In particular, the flat direction which has the largest VEV survives long. The longevity of the flat direction then ensures that thermalization of the inflaton decay products is considerably modified [4, 5].

The long lifetime of MSSM flat directions leads to late thermalization of inflaton decay products and a low reheat temperature. This gives rise to a natural solution to the thermal overproduction of gravitinos, and other dangerous relics [4, 5]. Regardless of their longevity, flat directions can also lead to a perturbative decay of a gauge singlet inflaton, even if it has large couplings to matter fields [8]. To summarize, the MSSM flat directions in general play crucial role in reheating and thermalization.

\section{ACKNOWLEDGMENTS}

The authors wish to thank Asko Jokinen, Keith Olive and Marco Peloso for useful discussions. The work of R.A. is supported by the National Sciences and Engineering Research Council of Canada (NSERC).
[1] M. Dine, L. Randall and S. Thomas, Phys. Rev. Lett. 75, 398 (1995). M. Dine, L. Randall and S. Thomas, Nucl. Phys. B 458, 291 (1996).

[2] T. Gherghetta, C. Kolda and S. P. Martin, Nucl. Phys. B 468, 37 (1996).

[3] For reviews, see K. Enqvist and A. Mazumdar, Phys. Rept. 380, 99 (2003); M. Dine and A. Kusenko, Rev. Mod. Phys. 76, 1 (2004).

[4] R. Allahverdi and A. Mazumdar, JCAP 0610, 008 (2006).

[5] R. Allahverdi and A. Mazumdar, arXiv:hep-ph/0505050.

[6] S. Davidson and S. Sarkar, JHEP 0011, 012 (2000).

[7] R. Allahverdi, Phys. Rev. D 62, 063509 (2000); R. Allahverdi and M. Drees, Phys. Rev. D 66, 063513 (2002); P. Jaikumar and A. Mazumdar, Nucl. Phys. B 683, 264 (2004).
[8] R. Allahverdi and A. Mazumdar, arXiv:hep-ph/0603244.

[9] R. Allahverdi, K. Enqvist, J. Garcia-Bellido and A. Mazumdar, Phys. Rev. Lett. 97, 191304 (2006).

[10] R. Allahverdi, A. Kusenko and A. Mazumdar, arXiv:hep-ph/0608138

[11] A. Jokinen and A. Mazumdar, Phys. Lett. B 597, 222 (2004).

[12] R. Allahverdi, K. Enqvist, A. Jokinen and A. Mazumdar, JCAP 0610, 007 (2006).

[13] K. Enqvist, S. Kasuya and A. Mazumdar, Phys. Rev. Lett. 90, 091302 (2003); K. Enqvist, A. Jokinen, S. Kasuya and A. Mazumdar, Phys. Rev. D 68, 103507 (2003);

[14] A. L. Maroto and A. Mazumdar, Phys. Rev. Lett. 84, 1655 (2000).

[15] R. Allahverdi, M. Bastero-Gil and A. Mazumdar, Phys. Rev. D 64, 023516 (2001); H. P. Nilles, M. Peloso and L. 
Sorbo, Phys. Rev. Lett. 87, 051302 (2001); H. P. Nilles, M. Peloso and L. Sorbo, JHEP 0104, 004 (2001).

[16] K. A. Olive and M. Peloso, Phys. Rev. D 74, 103514 (2006).

[17] J. Traschen and R. Brandenberger, Phys. Rev. D 42, 2491 (1990).

[18] L. Kofman, A. D. Linde and A. A. Starobinsky, Phys. Rev. D 56, 3258 (1997).

[19] M. Kawasaki, K. Kohri and T. Moroi, Phys. Lett. B 625, 7 (2005); M. Kawasaki, K. Kohri and T. Moroi, Phys. Rev. D 71, 083502 (2005).
[20] R. Allahverdi, R. H. A. Shaw and B. A. Campbell, Phys. Lett. B 473, 246 (2000).

[21] M. Postma and A. Mazumdar, JCAP 0401, 005 (2004).

[22] K. Enqvist, A. Jokinen and A. Mazumdar, JCAP 0401 , 008 (2004).

[23] H. E. Haber and G. L. Kane, Phys. Rept. 117 (1985) 75.

[24] R. Allahverdi and B. A. Campbell, Phys. Lett. B 395 , 169 (1997).

[25] T. Prokopec and T. G. Roos, Phys. Rev. D 55, 3768 (1997). 